\title{
Bone strength index in adolescent girls: does physical activity make a difference?
}

\author{
D A Greene, G A Naughton, J N Briody, A Kemp, H Woodhead, L Corrigan
}

Br J Sports Med 2005;39:622-627. doi: 10.1136/bjsm.2004.014498

See end of article for authors' affiliations

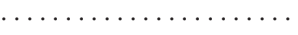

Correspondence to: Dr Greene, Australian Catholic University, Locked Bag 2002, Strathfield, NSW 2135, Australia; d.greene@mary.acu. edu.au

Accepted

27 September 2004

\begin{abstract}
Background: Bone strength index (BSI) combines bone mineral and bone biomechanical properties to measure resistance to bending. This index may have greater clinical significance than the more often described markers of bone mineral content (BMC), areal density, or geometry alone and, in turn, may show a stronger relation to fracture risk. The BSI is the product of volumetric cortical bone mineral density (BMD) and cross sectional moment of inertia within a region of interest. Calculations combine dual energy $x$ ray absorptiometry and magnetic resonance imaging technologies and provide a useful, non-invasive measure of in vivo bone strength.

Objectives: (a) To compare BSI in adolescent female middle distance runners and age matched controls; (b) to examine factors predictive of BSI in adolescent girls.

Methods: Twenty adolescent female middle distance runners (mean (SD) age 16 (1.7) years, physical activity 8.9 (2.1) hours a week) and 20 female controls (age 16 (1.8) years, physical activity 2.0 (0.07) hours a week) were recruited. To calculate BSI, a region of interest representing $10 \%$ of the middistal tibia was analysed for dual energy $x$ ray absorptiometry derived BMC and was combined with bone geometry and biomechanical properties obtained by magnetic resonance imaging assessments. Potential predictors of BSI were also examined.

Results: Independent $t$ tests showed that BMC ( $p=0.028)$, cortical bone volume $(p=0.002)$, volumetric cortical BMD $(p=0.004)$, cross sectional moments of inertia $(p=0.005)$, and BSI $(p=0.002)$ were higher in the distal tibia of athletes than of controls. The strongest predictor of BSI was hours of physical activity a week $\left(R^{2}=0.46\right)$.

Conclusions: Athletes habitually exposed to high training loads displayed greater BSI at the distal tibia than controls. The results further confirm BSI as a significant and discerning marker in musculoskeletal health in adolescent girls engaged in high and low mechanical loading.
\end{abstract}

i ifetime skeletal health is strongly influenced by acquisition of bone mass and geometry during childhood and -adolescence. Mechanical loading associated with physical activity enhances bone acquisition to a much greater extent in the developing ${ }^{1}$ than the mature skeleton. ${ }^{2}$ Optimising peak bone mass through physical activity during childhood and adolescence is a commonly cited strategy for reducing fracture risk in adult life. ${ }^{3}$

In long bones, cross sectional moment of inertia (CSMI) is a biomechanical indicator of the structural distribution of bone mass from the neutral bending axis. During growth, bone can respond to increased mechanical loading by geometric adaptation. Increases in bone apposition at periosteal and endosteal surfaces result in considerable increases in CSMI, ${ }^{4}$ with bone accumulated further from the neutral axis conferring a greater contribution to bone bending strength. ${ }^{5}$ Reports of CSMI and bone bending strength in child ${ }^{6}$ and adolescent ${ }^{7}$ sporting populations are scarce.

Bone mineral density (BMD) and bone geometry are strongly associated with ultimate bone strength. However, the positive effect of mechanical loading on bone geometry can be underestimated by dual energy $x$ ray absorptiometry (DEXA) measurements. ${ }^{8}$ An inability to accurately assess bone geometry from uniplanar DEXA derived data substantially detracts from DEXA based estimates of bone strength. ${ }^{9}$ Previously ${ }^{10}{ }^{11}$ bone strength was examined from peripheral quantitative computed tomography, but participant exposure to undesirable levels of radiation is of considerable concern. In animal research, bone strength index (BSI), obtained by integrating peripheral quantitative computed tomography and DEXA data, was found to correlate more closely with actual fracture load than either BMD or CSMI alone. ${ }^{12}$ Recently, cortical volumetric BMD and CSMI obtained from non-ionising magnetic resonance imaging (MRI) were combined with DEXA derived bone mineral content (BMC) to provide a useful, non-invasive measure of in vivo bone strength. Results showed that female adolescent athletes involved in weight loaded sport (running) had greater BSI at the mid-femur than athletes engaged in weight supported sports (swimming and cycling) and non-active controls. ${ }^{7}$ Despite the tibia being a commonly reported site of injury in middle distance runners, ${ }^{13}$ comparison of BSI at the distal tibia in highly trained female adolescent middle distance runners with non-active controls has not been reported. Habitual loading of the distal tibia may involve exposure to a combination of compressive and bending forces in elite adolescent middle distance runners.

The primary purpose of this study therefore was to compare distal tibial BMC, volumetric cortical BMD, CSMI, and BSI in elite level adolescent female middle distance runners with age matched controls. The secondary purpose was to examine factors predictive of BSI from dependent and independent variables including extensor and flexor muscle cross sectional area (CSA), lower limb muscular strength,

\footnotetext{
Abbreviations: $B M C$, bone mineral content; $B M D$, bone mineral density; BSI, bone strength index; CSMI, cross sectional moment of inertia; CSA, cross sectional area; CV, coefficient of variation; DEXA, dual energy $x$ ray absorptiometry; LTM, lean tissue mass; MRI, magnetic resonance imaging; $\mathrm{ROI}$, region of interest
} 

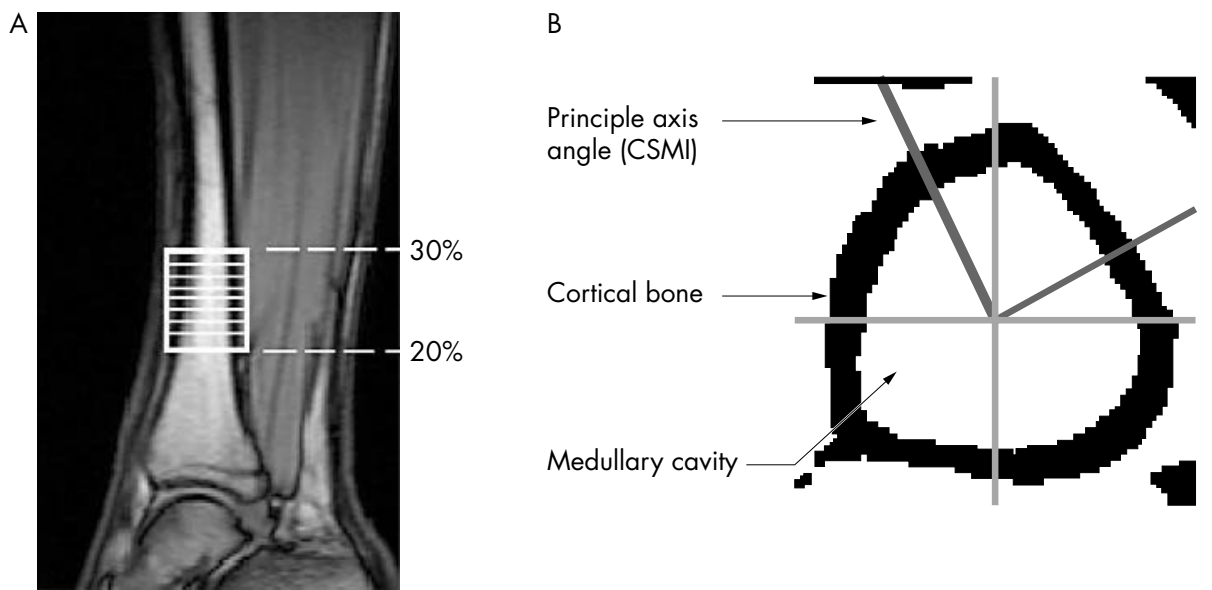

Figure 1 (A) Coronal scan of distal tibia showing 10\% region of interest (ROI); (B) cross sectional view of distal tibia from ROI showing cross sectional moment of inertia (CSMI) principle axis.

calcium intake, body composition profiles, oestrogen concentrations, and hours of physical activity a week.

\section{METHODS}

\section{Study participants}

Forty female adolescents (aged 13-18 years) comprised two groups of middle distance runners $(\mathrm{n}=20)$ and age matched controls $(\mathrm{n}=20)$. Eligibility criteria included athletes who had averaged a minimum of six hours a week of training for the previous two years, at an elite level (state or national representation). Those in the control group had completed less than three hours a week of physical activity. Participants were white and in good health, with no recent (previous two years) hospital admissions, history of illness lasting more than two weeks, or coexistent disease or drug use that could interfere with bone parameters. Approval was obtained from the ethics committees of the Australian Catholic University and The Children's Hospital at Westmead, in Sydney. An investigator (DG) explained procedures and requirements before parents and participants provided written consent.

\section{Procedures}

Testing involved a three hour visit to the Children's Hospital.

Table 1 Descriptive and modifiable lifestyle characteristics of adolescent female athletes and age matched controls ( $n=20$ per group)

\begin{tabular}{|c|c|c|c|}
\hline & Athlete & Control & p Value \\
\hline Age (years) & $15.9(1.6)$ & $16(1.8)$ & 0.894 \\
\hline Height $(m)$ & $1.66(0.5)$ & $1.62(0.6)$ & 0.03 \\
\hline Axial length $(\mathrm{cm})$ & $59.48(2.9)$ & $59.38(2.6)$ & 0.913 \\
\hline Femur length $(\mathrm{cm})$ & $43.3(1.8)$ & $42.02(1.8)$ & 0.031 \\
\hline Tibia length $(\mathrm{cm})$ & $35.3(1.9)$ & $34.1(1.8)$ & 0.063 \\
\hline Weight $(\mathrm{kg})$ & $52.2(6.1)$ & $58.7(10.8)$ & 0.027 \\
\hline BMI $\left(\mathrm{kg} / \mathrm{m}^{2}\right)$ & $18.7(1.5)$ & $22.2(4.1)$ & 0.002 \\
\hline Fat mass $(\mathrm{kg})$ & $8.2(2.8)$ & $19.5(8.4)$ & 0.001 \\
\hline Lean tissue $(\mathrm{kg})$ & $41.8(4.2)$ & $36.3(3.3)$ & 0.001 \\
\hline Plantar flexion (N.m) & 46.7 (11.1) & $46.3(16.3)$ & 0.928 \\
\hline Dorsiflexion (N.m) & $18.2(5.2)$ & $18.5(8.9)$ & 0.881 \\
\hline Physical activity (hours/ & & & \\
\hline week) & $8.9(4.1)$ & $2(0.7)$ & 0.001 \\
\hline Calcium (mg) & $1271.7(122)$ & $698.9(79.4)$ & 0.007 \\
\hline Age at menarche (years) & $12.5(4.3)$ & $\begin{array}{l}12.4(3.1) \\
152.20\end{array}$ & 0.953 \\
\hline Oestrogen (pmol/l) & $146.95(36.20)$ & $(43.60)$ & 0.862 \\
\hline
\end{tabular}

\section{Anthropometry}

Body weight was measured using electronic scales accurate to 500 g (UW150; Wedderburn, Sydney, Australia), and standing height was measured to $0.1 \mathrm{~cm}$ using a standard stadiometer (UW150; Wedderburn). Axial, femoral, and tibial lengths were determined by DEXA (Prodigy; Lunar Radiation Corp, Madison, Wisconsin, USA). From a total body scan, the ruler function was used to measure axial length from skull base to symphysis pubis. Femoral length was measured from femoral head midpoint to the base of the femoral condyle, and tibia length was the distance between the proximal, medial tibial plateau, and the base of the medial malleolus. The coefficient of variation (CV) for measured sites ranged between $0.3 \%$ and $1.3 \%$ for the same investigator (DG).

\section{Muscle strength}

Strength was measured using a Cybex Norm isokinetic dynamometer (Lumex Inc, Ronkonkoma, New York, USA). Plantar flexion and dorsiflexion torque (N.m) of the dominant foot were measured at $60 \%$, after standard positioning and stabilising procedures for the torso, legs, and ankle. Peak torque values from five maximal continuous flexion and extension repetitions were used as the criterion strength measure.

\section{Body composition and BMC}

Total body fat and lean tissue mass (LTM) were measured using DEXA. A spine scan (thin) was used to measure distal tibial BMC (g). A region of interest (ROI) box was positioned distally between $20 \%$ and $30 \%$ of tibial length, using custom analysis. Distal tibial BMC CV ranged between $0.4 \%$ and $1.6 \%$

Table 2 Bone mineral content (BMC), bone mineral density (BMD), and volumes representing 10\% distal tibial region of interest for female adolescent athletes and age matched controls ( $n=20$ per group)

\begin{tabular}{llll}
\hline & Athletes & Controls & $\mathrm{p}$ Value \\
\hline BMC (g) & $8.9(1.5)$ & $8(0.9)$ & 0.028 \\
$\begin{array}{l}\text { Cortical bone volume } \\
\left(\mathrm{cm}^{3}\right)\end{array}$ & $81.6(20.8)$ & $63.7(11.5)$ & 0.002 \\
$\begin{array}{l}\text { Volumetric cortical BMD } \\
\left(\mathrm{g} / \mathrm{cm}^{3}\right)\end{array}$ & & & \\
\hline
\end{tabular}

Values are mean (SD). 
for the same investigator (DG). The Lunar Prodigy CVs for total body fat and LTM were $<1.0 \%$.

\section{Bone and muscle geometry}

Bone geometry of the distal third tibia was measured using an MRI unit (1.5 T; Intera; Phillips, Best, the Netherlands). Participants placed their dominant leg in a manufacturer supplied knee coil for about 30 minutes. The distal third demarcation between the medial malleolus and the tibial tuberosity was located using a coronal scout scan. Contiguous transverse images of the distal third region were then acquired (Tl weighted spin echo, TR 525 milliseconds, TE 15 milliseconds, $60 \times 3 \times 0.3 \mathrm{~mm}$ ). The image corresponding to $25 \%$ of tibial length was used as a reference point from which a $10 \%$ ROI was analysed (fig 1). Tibial cortical and medullary CSA $\left(\mathrm{mm}^{2}\right)$ were measured for each slice over the entire 10\% ROI, using Analyze software (Mayo Foundation, Rochester, Minnesota, USA; version 7.0) autotrace function. Individual tibial length determined the number of slices analysed. Volumetric measurements were calculated as the summed products of cortical CSA and slice thicknesses over the $10 \%$ ROI. Extensor, flexor, and total muscle CSA $\left(\mathrm{mm}^{2}\right)$ were measured at the mid slice of the $10 \%$ ROI. Intrainvestigator CSA CVs ranged from $0.6 \%$ to $4 \%(n=40)$.

Biomechanical variables

CSMIs were calculated using Scion software (Frederick, Maryland, USA; version Beta 3B) and a customised algorithm. Analyses involved the distal, mid, and proximal slices of the $10 \%$ ROI. The average principle axis value of the three slices was used to calculate an average CSMI over the entire $10 \%$ ROI. $^{7}$ Intrainvestigator CSMI CV was $0.6-1 \%(\mathrm{n}=15)$.

\section{Bone strength index}

BSI of the $10 \%$ ROI was determined using a previously published equation ${ }^{12}$ :

BSI $=$ CSMI $\times$ volumetric cortical BMD

DEXA derived distal tibial BMC (g) was divided by MRI derived distal tibial cortical bone volume $\left(\mathrm{cm}^{3}\right)$ to obtain volumetric cortical BMD $\left(\mathrm{g} / \mathrm{cm}^{3}\right)$. Thus BSI quantifies both the accumulation of bone mass and its distribution within bone.

\section{Pubertal status, menstrual history, and oestrogen} concentration

Pubertal status was determined using self reported assessment of Tanner stage for pubic hair and breast development. ${ }^{14}$ Menstrual history was determined by a questionnaire that included age at menarche and number of menses in the previous 12 months. Menses was coded into three categories; premenarcheal, age related oligomenorrhoea (onset of menarche within past six months), and eumenorrhoea (more than nine cycles a year). ${ }^{15}$ Oestrogen concentrations were measured using an ultrasensitive assay ( $\mathrm{pmol} / \mathrm{l}$ ) with an intra-assay $\mathrm{CV}$ of $<3.0 \%$ from samples of blood collected on the day of testing. Testing did not coincide with stage of menstrual cycle.

\section{Physical activity and injury history}

Duration and type of physical activity were estimated using Bouchard's physical activity record. ${ }^{16}$ Participants completed a medical and injury history questionnaire. Medical treatment, drug use, and number of weeks absent from training and/or competition during the previous 12 months were included from retrospective recall.

\section{Intake of macronutrients and calcium}

A three day food diary was completed by participants for two week days and one weekend day. Dietary analysis used Foodworks software version 2.1 (Xyris Software, Highgate Hill, Queensland, Australia).

\section{Statistical analysis}

After tests for normality, independent $t$ tests determined between-group differences. Relations between BSI, selected descriptive statistics, and other variables were determined by bivariate correlation analysis (Pearson correlation coefficient) for combined groups. Multiple linear regression assessed the contribution of strongly correlated variables to BSI. Analysis of covariance determined the independent effects of LTM and fat mass on group differences in BSI. Group sample size was based on observed effect sizes for site specific measures of areal $\mathrm{BMD}\left(\mathrm{g} / \mathrm{cm}^{2}\right)$ ranging from 0.5 to $2.0 \mathrm{SD}$ in cross sectional studies involving healthy adolescents. ${ }^{17}{ }^{18}$ A sample size of 20 participants in the athlete and control groups was expected to detect significant differences at $\mathrm{p}<0.05$ with a statistical power of $80 \%$. Statistical analysis used SPSS version 11.5 (SPSS Inc, Chicago, Illinois, USA).

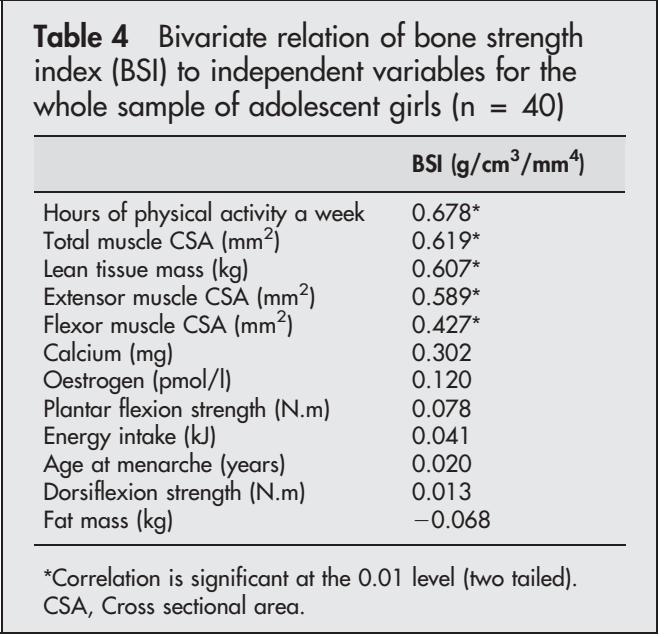

Table 3 Distal tibial bone and muscle geometry and biomechanical variables

\begin{tabular}{llll}
\hline & Athlete & Control & p Value \\
\hline Total CSA $\left(\mathrm{mm}^{2}\right)$ & $363.18(31.4)$ & $364.4(43.7)$ & 0.76 \\
Cortical CSA (mm $\left.{ }^{2}\right)$ & $195.29(32.9)$ & $158.05(23.8)$ & 0.005 \\
Medullary cavity CSA $\left(\mathrm{mm}^{2}\right)$ & $165.7(56.5)$ & $206.2(51.5)$ & 0.023 \\
CSMI $\left(\mathrm{mm}^{4}\right)$ & $10039.82(2105.6)$ & $8041.76(2080.1)$ & 0.005 \\
$\mathrm{BSI}\left(\mathrm{g} / \mathrm{cm}^{3} / \mathrm{mm}^{4}\right)$ & $92950.3(30545.6)$ & $64912.8(20410.5)$ & 0.002 \\
Total muscle CSA $\left(\mathrm{mm}^{2}\right)$ & $1695.3(428.03)$ & $1455.2(227.8)$ & 0.033 \\
Extensor muscle CSA $\left(\mathrm{mm}^{2}\right)$ & $373.8(70.7)$ & $326.9(51.4)$ & 0.021 \\
Flexor muscle CSA (mm $)$ & $419.5(36.5)$ & $304(41.6)$ & 0.135 \\
\hline Values are mean (SD). & & & \\
BSI, Bone strength index; CSMl, cross sectional moment of inertia; CSA, cross sectional area. & \\
\hline
\end{tabular}




\section{RESULTS}

\section{Descriptive characteristics}

Table I summarises the descriptive characteristics of the participants. Compared with non-athletic controls, athletes had lower weight (mean (SD) $-6.47(2.7) \mathrm{kg}, \mathrm{p}=0.027$ ), body mass index $\left(-3.45(0.9) \mathrm{kg} / \mathrm{m}^{2}, \mathrm{p}=0.002\right)$, and fat mass $(-11.3(1.9) \mathrm{kg}, \mathrm{p}=0.001)$. Athletes were also taller $(+3.9(1.7) \mathrm{cm}, \mathrm{p}=0.030)$, consumed more calcium (+572.7 (201.2) mg, p = 0.007), had greater LTM $(+5.48$ (1.2) kg, $\mathrm{p}=0.001)$, and engaged in more hours of physical activity a week $(+6.9$ (1.07) hours, $p=0.001)$ than non-athletic controls. The greater height in athletes compared with controls was explained by greater femoral length $(\mathrm{p}=$ $0.03)$, not tibial $(p=0.06)$ or axial length $(p=0.91)$. No between-group differences were observed for age, plantar flexion or dorsiflexion torque at the ankle, age at menarche, menstrual status, or oestrogen concentration $(\mathrm{p}>0.05)$. Menstrual status in athletes showed categorical distributions of $10 \%$ premenarcheal, $30 \%$ with age related oligomenorrhoea and $60 \%$ with eumenorrhoea. Similarly, the relative distribution for the three categories of menstrual status in the control group was $8 \%$ premenarcheal, $30 \%$ with age related oligomenorrhoea and $62 \%$ eumenorrhoea. Pearson $\chi^{2}$ analysis showed no differences in menstrual status between groups $(\mathrm{p}>0.05)$.

\section{Bone mineral properties}

Table 2 presents absolute values for distal tibial BMC, BMD, and cortical volumetric BMD. Athletes displayed greater BMC $(+0.95(0.4) \mathrm{g}, \mathrm{p}=0.028), \mathrm{BMD}\left(+17.88(5.3) \mathrm{cm}^{3}, \mathrm{p}=\right.$ $0.002)$, and volumetric density $\left(+1.18(0.54) \mathrm{g} / \mathrm{cm}^{3}, \mathrm{p}=\right.$ 0.034 ) at the $10 \%$ ROI than controls.

\section{Geometry and biomechanical and bone strength variables}

Table 3 shows total, cortical, and medullary CSA, CSMI, BSI, and total muscle, extensor, and flexor CSA for the entire $10 \%$ ROI. Athletes had greater tibial cortical bone (+27.23 (9.09) $\mathrm{mm}^{2}, \mathrm{p}=0.005$ ) and smaller medullary cavity CSA $\left(-40.41(17.11) \mathrm{mm}^{2}, \mathrm{p}=0.023\right)$ than non-athletes. Athletes and non-athletes, however, were not different in total tibial bone CSA $(\mathrm{p}>0.05)$. Athletes also showed greater CSMI $\left(+1998.05(661.8) \mathrm{mm}^{4}, \mathrm{p}=0.005\right)$ and BSI $\left(+28037.48(8214.7) \mathrm{g} / \mathrm{cm}^{3} / \mathrm{mm}^{4}, \mathrm{p}=0.002\right)$ than nonathletes. Total muscle $\left(+240.2(86.4) \mathrm{mm}^{2}, \mathrm{p}=0.033\right)$ and extensor muscle CSA $\left(+46.9(19.5) \mathrm{mm}^{2}, \mathrm{p}=0.021\right)$, but not flexor muscle CSA $(\mathrm{p}>0.05)$, were greater in athletes than controls. Figure 1 illustrates distal tibial ROI and CSMI.

Table 4 presents bivariate correlations between measures of BSI and selected descriptive and independent variables. Hours of physical activity a week, total muscle CSA, LTM, and extensor and flexor muscle CSA correlated with BSI $(p<0.01)$. No other significant correlations between BSI and selected descriptive variables emerged. Multiple regression analysis was used to determine the strongest predictive factors of variability in BSI. Hours of physical activity a week, total muscle CSA, and LTM explained 58\% of the variance in BSI for combined groups $(n=40)$. The regression equation was:

BSI $=-24992.4+(2635.03 \times$ hours of physical activity a week $)+(20.03 \times$ total muscle CSA $)+(1.502 \times$ LTM $)$

giving $R^{2}=0.58$. Analysis of covariance was used to adjust for differences in LTM and fat mass between athletes and controls. Group mean differences in BSI were reported after covariation for fat mass $(p=0.011)$ but not LTM $(p>0.05)$.

\section{DISCUSSION}

This is the first study to report differences in structural and material bone properties between two groups of adolescent girls exposed to different mechanical loading. Specifically, athletes displayed greater BSI, CSMI, cortical bone CSA, total and extensor muscle CSA, volumetric cortical BMD, and BMC at the distal tibia. They also displayed smaller medullary cavity CSA than controls, but the two groups did not differ in total tibial CSA or tibial length. When BSI data were controlled for fat mass, greater BSI in athletes remained. However, no between-group differences in BSI were found after covariation for LTM. Body composition profiles of athletes showed greater LTM and less fat mass than controls.

To our knowledge, only two studies ${ }^{70}$ have combined MRI with DEXA derived data to examine bone strength in children and adolescents. Loaded versus non-loaded humeral bone strength in prepubertal, peripubertal, and postpubertal female tennis players revealed a proportional increase in periosteal apposition and endocortical resorption versus the contralateral limb only in prepubertal athletes. Cortical bone further from the neutral axis increased humeral resistance to bending, so the observed growth related changes made the bones of prepubertal tennis players stronger. Loading associated with the postpubertal years, however, increased endocortical apposition and contributed less to humeral bone strength. ${ }^{20}$ In the present study, greater endocortical apposition in athletes was also apparent in the distal tibia of athletes, despite similar total tibial CSA in the two groups. Increased cortical thickness and a narrowing of the medullary cavity, however, promoted bone strength in athletes, as evidenced by greater CSMI than the controls. Although endocortical apposition confers a smaller biomechanical advantage than periosteal apposition, greater cortical thickness still confers a biomechanical advantage and may protect against future age related endocortical resorption. ${ }^{1}$

In the present study, the number of hours of physical activity a week was the strongest predictor of BSI. Our results add to previous intervention and cross sectional studies assessing the effects of physical activity on bone strength in children, ${ }^{146}$ adolescents, ${ }^{7}$ and young adults. ${ }^{811}$ No previous studies have examined the link between physical activity and BSI at the distal tibia. Impact forces and training intensity associated with middle distance running were not measured in this study. However, athletes engaged in four times the number of hours of physical activity a week than controls, and activity accounted for over $57 \%$ of the variance in BSI.

Bone strength in adolescent female athletes engaged in either weight bearing or non-weight bearing sports has also been examined at the mid-femur using a three dimensional technique. $^{7}$ Results revealed a positive association between mid-femur bone strength and weight bearing activity. Differences in BSI among athletic groups, however, were associated with differences in CSMI (geometry) and not bone material, with cortical volumetric BMD similar between the groups. In contrast, the bone material of athletes in our study showed greater cortical volumetric BMD than non-athletic controls as well as advantages in geometry, despite similar tibial lengths. In addition to bending forces experienced at the distal tibia, ${ }^{21}$ considerable axial, compressive loading may be inherent in training for middle distance running in female adolescent populations. Compressive loads may provide a greater osteogenic stimulus for accumulation of bone material than forces experienced at other sites in the body and in other weight bearing sports.

DEXA based hip structural analyses ${ }^{4}{ }^{6}$ have previously described influences of mechanical loading and bone strength. In this study, assumptions of cylindrical bone shape and estimates of cortical thickness inherent in DEXA based calculations were not necessary. MRI enabled accurate measurement of bone compartment CSA and volume. By combining MRI with BMC from DEXA, between-group 


\section{What is already known on this topic}

- Skeletal health is strongly influenced by acquisition of bone mass and geometry during childhood and adolescence.

- Animal research has shown that $B S I$ is a better measure of fracture load than variables such as BMD.

- Female adolescent athletes involved in weight loaded sport (running) have greater BSI at the mid-femur than athletes engaged in weight supported sports (swimming and cycling) and non-active controls.

\section{What this study adds}

- Athletes exposed to high training loads display greater BSI at the distal tibia than controls.

- BSI is confirmed as a discerning marker of musculoskeletal health.

differences in true cortical volumetric BMD for the entire mid-distal tibial region were precisely quantified.

Results from this study also support the muscle-bone unit paradigm. ${ }^{22}$ A functional muscle-bone relation is based on the assumption that loading effects on muscle also influence bone. Muscle forces place greater loads on bones than gravitational forces associated with weight bearing activity. As muscle strength correlates with muscle mass, bone strength, theoretically, should correlate with muscle CSA and LTM. ${ }^{6}$ Previous studies, ${ }^{22-24}$ however, have examined the effect of muscle force on surrogates of bone strength, such as BMC and BMD, rather than the combined influence of bone material and structural properties. In this study, the positive influence of LTM on BSI was evident after covariation for fat mass, with athletes displaying greater mid-distal tibial BSI. In contrast, when the influence of LTM on BSI was controlled, between-group differences were not evident. Furthermore, total muscle CSA, LTM, and extensor and flexor muscle CSA were strong correlates of BSI. Our results support previous findings of moderate to strong site specific correlations in lower limb bone strength with muscle CSA in young adults. ${ }^{21}$ Further investigation of the muscle-bone unit is needed using bone strength, rather than BMD as an outcome variable among adolescent sporting populations.

The cross sectional design of this study cannot discount the potential influence of selection bias, genetics in bone material and structural outcomes, and even trainability among groups. Furthermore, bone adaptation to mechanical loading is site specific. Assessment of an ROI at the distal tibia does not represent the entire tibial response to mechanical loading. ${ }^{19}$ Assessment of bone strength in middle distance runners at additional sites exposed to bending and compressive loads would advance our understanding of total body musculoskeletal adaptations in adolescent sporting populations.

In conclusion, this study compared bone material and structural properties in elite adolescent female middle distance runners with age matched controls. Athletes displayed greater BSI, CSMI, cortical bone CSA, total muscle and extensor muscle CSA, volumetric cortical BMD, and BMC at the distal tibia than non-athletes. Similarly, total muscle and extensor muscle CSA were greater in athletes than controls and contributed to variability in BSI. Importantly, our findings show a positive association between hours of physical activity a week, and tibial bone material (volumetric cortical BMD) and bone biomechanical indices (BSI and CSMI) of musculoskeletal health in adolescent girls involved in middle distance running. It appears that physically active adolescent girls are advantaged in bone strength at the distal tibia compared with non-active controls.

\section{ACKNOWLEDGEMENTS}

We thank the New South Wales Sporting Injuries Committee for their financial support to conduct the research, and Kenneth Graham and Rob Medlicott from the New South Wales Institute of Sport for assistance in recruiting athletic participants.

\section{Authors' affiliations}

D A Greene, G A Naughton, Centre of Physical Activity Across the Lifespan (CoPAAL), Australian Catholic University, Quakers Hill, NSW, Australia

J N Briody, Department of Nuclear Medicine, The Children's Hospital, Westmead, Australia

A Kemp, Department of Medical Imaging, The Children's Hospital, Westmead

H Woodhead, Department of Endocrinology, The Children's Hospital, Westmead

L Corrigan, Laurentian University, Sudbury, Ontario, Canada

Competing interests: none declared

\section{REFERENCES}

1 Bradney M, Pearce G, Naughton G, et al. Moderate exercise during growth in prepubertal boys: changes in bone mass, size, volumetric density and bone strength: a controlled prospective study. J Bone Miner Res 1998;13:1814-21.

2 Cavanaugh DJ, Cann CE. Brisk walking does not stop bone loss in postmenopausal women. Bone 1988;9:201-4.

3 Bailey DA, McKay HA, Mirwald RL, et al. A six-year longitudinal study of the relationship of physical activity to bone mineral accrual in growing children: the University of Saskatchewan Bone Mineral Accrual Study. J Bone Miner Res 1999; 14:1672-9.

4 Petit MA, McKay HA, MacKelvie KJ, et al. A randomized school-based jumping intervention confers site and maturity-specific benefits on bone structural properties in girls: a hip structural analysis study. J Bone Miner Res 2002; 17:363-72

5 Beck TJ, Oreskovic TL, Stone KL, et al. Structural adaptation to changing skeletal load in the progression toward hip fragility: the study of osteoporotic fractures. J Bone Miner Res 2001;16:1108-19.

6 Faulkner RA, Forwood MR, Beck TJ, et al. Strength indices of the proximal femur and shaft in prepubertal female gymnasts. Med Sci Sports Exerc 2003;35:513-18.

7 Duncan CS, Blimkie JR, Cowell CT, et al. Bone mineral density in adolescent female athletes: relationship to exercise type and muscle strength. Med Sci Sports Exerc 2002;4:286-94.

8 Haapasalo H, Kountulainen S, Sievanen H, et al. Exercise-induced bone gain is due to enlargement in bone size without a change in volumetric bone density: a peripheral quantitative computed tomography study of the upper arms of male tennis players. Bone 2000;27:351-7.

9 Woodhead HJ, Kemp AF, Blimkie CJR, et al. Measurement of mid-femoral shaft geometry: repeatability and accuracy using MRI and dual energy x-ray absorptiometry. J Bone Miner Res 2001;16:2251-9.

10 Kontulainen S, Sievanen $H$, Kannus $P$, et al. Effect of long-term impactloading on mass, size, and estimated strength of humerus and radius of female racquet-sports players: a peripheral quantitative computed tomography study between young and old starters and controls. J Bone Miner Res 2003; 18:352-9.

11 Heinonen, Sievanen, Kannus, et al. Site-specific response to long-term weight training seems to be attributable to principal loading modality: a PQCT study
of female weightlifters. Calcif Tissue Int 2002;70:469-74.

12 Ferretti JL, Capozza RF, Zanchetta JR. Mechanical validation of a tomographic (PQCT) index for noninvasive estimation of rat femur bending strength. Bone 1996;18:97-102.

13 Bennell KL, Crossley K. Musculoskeletal injuries in track and field: incidence, distribution and risk factors Aust J Sc Med Sport 1996;28:69-75.

14 Tanner TM. In: Growth at adolescence, 2nd ed. Oxford: Blackwell Scientific Publications, 1968:167-205.

15 Fielsler CM. The female runner. In: O'Connor FG, Wilder RP, eds. Textbook of running medicine. New York: McGraw Hill, 2001:435-46.

16 Bouchard C, Tremblay A, Leblanc C, et al. A method to assess energy expenditure in children and adults. Am J Clin Nut 1983;37:461-7.

17 Duncan CS, Blimkie CJ, Cowell CT, et al. Bone mineral density in adolescent female athletes: relationship to exercise type and muscle strength. Med Sci Sports Exerc 2002;34:286-94

18 Nichols DL, Sanborn CF, Bonnick SL, et al. The effects of gymnastics training on bone mineral density. Med Sci Sports Exerc 1994;26:1220-5. 
19 Forwood MR. Mechanical effects on the skeleton: are there clinical implications? Osteoporos Int 2001;12:77-83.

20 Bass SL, Saxon L, Daly RM, et al. The effect of mechanical loading on the size and shape of bone in pre-, peri-, and postpubertal girls: a study in tennis players. J Bone Miner Res 2002;17:2274-80.

21 Rittweger J, Beller G, Ehrig J, et al. Bone-muscle strength indices for the human lower leg. Bone 2000;27:319-26.

22 Schoenau E, Frost HM. The "muscle-bone unit" in children and adolescents. Calcif Tissue Int 2002;70:405-7.

23 Sandstrom $\mathbf{P}$, Jonsson $\mathrm{P}$, Lorentzon $\mathrm{R}$, et al. Bone mineral density and muscle strength in female ice hockey players. Int J Sports Med 2000;21:524-8.

24 Pettersson U, Nordstrom P, Alfredson $\mathrm{H}$, et al. Effect of high impact activity on bone mass and size in adolescent females: a comparative study between two different types of sports. Calcif Tissue Int 2001;67:207-14.

\section{COMMENTARY}

The most compelling evidence for a beneficial effect of exercise on bone mineral density (BMD) is found during growth. Prospective, controlled studies have shown that even moderate exercise increases BMD during growth, predominantly during the prepubertal and peripubertal years.
Furthermore, high impact loading in gymnastics, soccer, weightlifting, and racket sports is associated with an even higher regional BMD. The higher BMD is the result of surface specific periosteal modelling increasing bone size and endosteal (endocortical, trabecular, intracortical) surface modelling and remodelling producing thicker cortices, all changes of importance for bone strength. Whether the cortices are less porous, because of fewer or smaller haversian canals, is uncertain as is whether exercise results in thicker or more trabeculae. In addition, the changes in the skeleton may be sex, maturation, and region specific related to physical activity, and also different types of physical activity may provoke different skeletal responses. This study highlights, by use of magnetic resonance imaging and dual energy $x$ ray absorptiometry, not only the changes in BMD with exercise but also structural changes in the skeleton of the lower extremity.

M Karlsson

Department of Orthopaedics, Malmö University Hospital, Lund University, SE-20502 Malmö, Sweden; magnus.karlsson@orto.mas.lu.se

\section{bmjupdates+}

bmiupdates+ is a unique and free alerting service, designed to keep you up to date with the medical literature that is truly important to your practice.

bmjupdates+ will alert you to important new research and will provide you with the best new evidence concerning important advances in health care, tailored to your medical interests and time demands.

Where does the information come from?

bmjupdates+ applies an expert critical appraisal filter to over 100 top medical journals A panel of over 2000 physicians find the few 'must read' studies for each area of clinical interest

Sign up to receive your tailored email alerts, searching access and more...

www.bmjupdates.com 